\title{
Cloud-Based Implementation of New Frontline Clinical Workflows: Standardizing Practice at Scale to Improve Patient Safety
}

Jennie Yoo ${ }^{1}, \mathrm{MD} ;$ Mary Han $^{1}$, MD; Gemma Jamena ${ }^{2}, \mathrm{MD}$; Phyllis Pei ${ }^{2}, \mathrm{RN} ;$ Hillary Baldocchi ${ }^{2}, \mathrm{PhD}$; Emilie Menard ${ }^{3}$, NP; Shelley Diane ${ }^{3}, \mathrm{RN}$; Peter Oishi ${ }^{4}, \mathrm{MD}$; Tam Nguyen ${ }^{2}, \mathrm{PhD}$

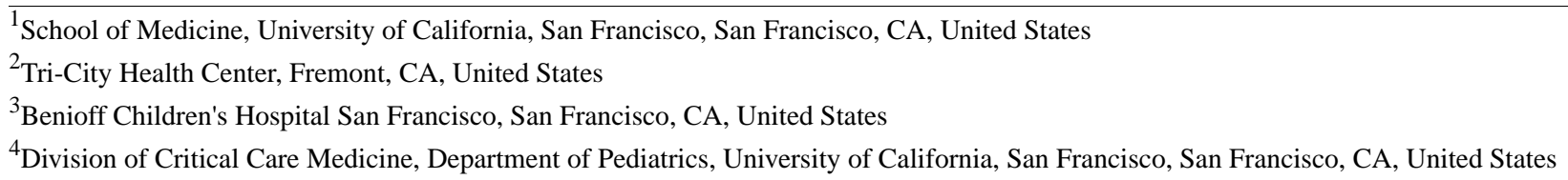

Corresponding Author:

Jennie Yoo, MD

School of Medicine

University of California, San Francisco

513 Parnassus Avenue

San Francisco, CA,

United States

Phone: 4762342

Email: jennie.yoo@ucsf.edu

\begin{abstract}
Background: Implementation of new practices in large health care settings is difficult. Staff are already overwhelmed, and practice deviation is common. With time-constrained visits, providers struggle to address complex problems. Three scenarios were identified where frontline practice standardization would improve patient outcomes: sedation and analgesia for intubated patients (inpatient), colorectal cancer screenings (outpatient), and safety measures for opioid prescriptions (outpatient). We implemented these practices through a cloud-based solution designed for frontline health care staff, fostering peer-accountability and transparency of processes.
\end{abstract}

Objective: 1) Introduce a standard approach to sedation and analgesia for intubated patients. 2) Increase colorectal cancer screenings for the clinic population. 3) Improve opioid safety for patients with chronic opioid use.

Methods: Practices were implemented through a cloud-based app (Elemeno Health, Oakland, CA) that allows frontline health care teams to access an organization's best practices through interactive decision guides, smart checklists, and how-to videos from any device. In a pediatric ICU, we first delivered a Critical Care Comfort Algorithm (CALM) for titrating sedative and analgesia medications, a bottom-up self-assessment for frontline staff to evaluate their performance, and a top-down audit checklist for charge nurses to complete. For multiple community health centers, we created colorectal cancer screening practice decision guides for medical assistants (MA) and providers, and deployed the practices through a 3-week gamified contest between individual clinics conducted through the app. For the opioid safety initiative, we created a Provider Chronic Pain Management Workflow checklist, Provider Pain Evaluation Guide, and a MA checklist for medication reconciliation; implementation was paired with a 2-month inter-clinic competition.

Results: Within 2 weeks of the formal roll-out of the Pediatric ICU charge nurse audit tool, 107 checklists were completed and $83 \%$ of intubated patients were on the sedation protocol. During the gamified 3 weeks for colorectal cancer screening, 2107 checklists were completed with engagement from $74 \%$ of MAs and $80 \%$ of providers. MAs appeared to habituate to the practice with ongoing practice post-competition; there was a $70 \%$ increase in colorectal cancer screenings 1 year post-intervention. During the contest period for increased opioid safety, naloxone prescription increased from $<10 /$ month to $27 /$ month for new prescriptions and $21 /$ month for renewals. Opioid contracts with historically negligible adherence increased to $45 / \mathrm{month}$ for new contracts and $53 /$ month for renewed contracts. There was also a $70 \%$ increase in referrals to the Behavioral Health Pain Management Program.

Conclusions: Our clinical improvement initiative using cloud-based real-time actionable and trackable decision guides facilitated staff engagement with standardized protocols for pediatric analgesia and sedation, led to a significant increase in colorectal cancer screenings with high levels of provider and staff participation, and improved opioid safety and utilization of behavioral support resources for patients with chronic opioid use. The cloud-based application empowers staff with just-in-time access to microlearning 
tools and resources to manage patient care, simplifying management's ability to train staff at scale. Standardizing practice and streamlining workflows liberalizes valuable face-to-face time with patients and improves patient safety.

(iproc 2018;4(2):e11776) doi: $10.2196 / 11776$

Edited by T Hale; this is a non-peer-reviewed article. Submitted 02.08.18; accepted 29.08.18; published 17.09.18.

Please cite as:

Yoo J, Han M, Jamena G, Pei P, Baldocchi H, Menard E, Diane S, Oishi P, Nguyen T

Cloud-Based Implementation of New Frontline Clinical Workflows: Standardizing Practice at Scale to Improve Patient Safety iproc 2018;4(2):e11776

URL: http://www.iproc.org/2018/2/e11776/

doi: $\underline{10.2196 / 11776}$

PMID:

(CJennie Yoo, Mary Han, Gemma Jamena, Phyllis Pei, Hillary Baldocchi, Emilie Menard, Shelley Diane, Peter Oishi, Tam Nguyen. Originally published in Iproceedings (http://www.iproc.org), 17.09.2018. This is an open-access article distributed under the terms of the Creative Commons Attribution License (https://creativecommons.org/licenses/by/4.0/), which permits unrestricted use, distribution, and reproduction in any medium, provided the original work, first published in Iproceedings, is properly cited. The complete bibliographic information, a link to the original publication on http://www.iproc.org/, as well as this copyright and license information must be included. 